\title{
Gestational Diabetes Mellitus: The Genetic Susceptibility Behind the Disease
}

Authors

Wenwen Wei, Yuejuan He, Xin Wang, Guiqin Tan, Fangyu Zhou, Guangbing Zheng, Dan Tian, Xiaomin Ma, Hongsong $\mathrm{Yu}$

\author{
Affiliation \\ School of Basic Medical Science, Special Key Laboratory of \\ Ocular Diseases of Guizhou Province, Zunyi Medical \\ University, Guizhou, Zunyi, China \\ Key words \\ gestational diabetes mellitus, single-nucleotide polymor- \\ phism, genetics, susceptibility \\ received 13.04.2021 \\ accepted after revision $\quad 30.06 .2021$ \\ Bibliography \\ Horm Metab Res 2021; 53: 489-498 \\ DOI 10.1055/a-1546-1652 \\ ISSN 0018-5043 \\ (C) 2021. Thieme. All rights reserved. \\ Georg Thieme Verlag KG, Rüdigerstraße 14, \\ 70469 Stuttgart, Germany \\ Correspondence \\ Hongsong Yu \\ School of Basic Medical Science \\ Zunyi Medical University \\ 6 Xuefu West Road \\ 563000 Guizhou \\ Zunyi \\ China \\ Tel.: + 8685128642292 \\ yuhongsong@163.com
}

\author{
丹 Supplementary material is available under https:// \\ doi.org/10.1055/a1546-1652
}

\begin{abstract}
Gestational diabetes mellitus (GDM), a type of pregnancy-specific glucose intolerance or hyperglycemia, is one of the most common metabolic disorders in pregnant women with $16.9 \%$ of the global prevalence of gestational hyperglycemia. Not only are women with GDM likely to develop T2DM, but their children are also at risk for birth complications or metabolic disease in adulthood. Therefore, identifying the potential risk factors for GDM is very important in the prevention and treatment of GDM. Previous studies have shown that genetic predisposition is an essential component in the occurrence of GDM. In this narrative review, we describe the role of polymorphisms in different functional genes associated with increased risk for GDM, and available evidence on genetic factors in the risk of GDM is summarized and discussed.
\end{abstract}

\section{Introduction}

Gestational diabetes mellitus (GDM), a type of pregnancy-specific glucose intolerance or hyperglycemia, is one of the most common metabolic disorders in pregnant women [1]. GDM has emerged as an urgent public health concern, with more than a $30 \%$ increase in prevalence over the past few decades [2]. According to the International Diabetes Federation, the global prevalence of gestational hyperglycemia is $16.9 \%$, including all cases of diabetes in pregnancy (i.e., known and previously undiagnosed diabetes) and gestational diabetes [3]. Epidemiologic studies have identified several GDM risk factors such as advanced maternal age, ethnicity, a previous history of gestational diabetes, and a family history of type 2 diabetes mellitus (T2DM) [4]. Offspring of mothers with GDM have an increased risk of birth complications associated with increased birth weight and adiposity - including shoulder dystocia, neonatal hypoglycemia, and obesity - as well as developing metabolic syndrome, T2DM, and cardiovascular disease in adult life. Furthermore, in a 5-10-year perspective, it was estimated that approximately $50 \%$ of the women diagnosed with GDM will develop T2DM [5]. Thus, it is of paramount clinical value to identify potential risk factors for GDM, and to provide evidence for early-prevention measures [2].

The occurrence of GDM is associated with a variety of cellular and functional disorders, of which both $\beta$-cell impairment and tis- 
sue insulin resistance represent critical components of the pathophysiology of GDM [6]. The pancreatic $\beta$-cells perform the functions of storing and secreting insulin in response to glucose load [7]. Deficiencies in $\beta$-cell machinery may appear during pregnancy, as these cells may not sense glucose concentration in the circulation effectively or generate enough insulin to maintain glucose homeostasis [7]. In addition, with the progression of pregnancy, the ability of insulin to bind to insulin receptors on the cell surfaces of peripheral tissues, such as skeletal muscles, diminishes [4], leading to insulin resistance. In general, pregnant women are physiologically hyperglycemic [7], and a hyperglycemic environment is associated with oxidative stress [6]. Oxidative stress can reduce glucose transporter type 4 (GLUT4) expressions by impairing nuclear protein transfer to the insulin-responsive element in the GLUT4 promoter to affect glucose transport efficiency [7]. The cell-signaling proteins that are secreted by adipose tissues are primarily adiponectin and leptin, which can then be used as control regulators of neurohormone metabolism to regulate appetite, active energy expenditure, and basal metabolic rate [6]. Dysfunction in this hormone-regulatory network can lead to adiposity and influence glucose utilization, thereby aggravating insulin resistance [6]. In summary, decreased insulin secretion caused by $\beta$-cell injury and dysfunction, peripheral insulin resistance, oxidative stress, and an imbalance in the neural hormone metabolic network collectively contribute to the occurrence of GDM.

Although multiple risk factors for GDM have been identified, the mechanism of action underlying these risk factors remains arcane. However, an increasing number of reports indicate that GDM carries a strong genetic susceptibility [2]. We searched the PubMed, Embase and Web of Science databases for association studies identifying the susceptibility gene of GDM. The key words were: "Gestational diabetes mellitus or GDM or gestational diabetes" and "gene polymorphism or single-nucleotide polymorphism or genetic variants". We found that in recent years, several genome-wide association studies (GWAS) and case-control studies have shown that gene polymorphism is significantly associated with susceptibility to GDM. Therefore, we objectively, accurately, and comprehensively summarized herein the relationship between gene single-nucleotide polymorphisms (SNPs) and GDM - including $\beta$-cell function, insulin secretion, insulin resistance, glucose metabolism, and oxidative stress.

\section{GWAS and GDM}

Several events have laid the foundation for GWAS, such as the completion of the human genome sequence, the storage of millions of SNPs in the public database, the rapid improvement of SNP genotyping technology, and the launch of the International HapMap Project [8]. GWAS can explore the roles of the most common genetic variants in disease and determine the heritable quantitative traits that are risk factors for disease [8]. A GWAS, involving 4437 pregnant women of European, Thai, Afro-Caribbean, and Hispanic heritage, showed that SNPs of glucose kinase regulator (GCKR) (rs1260326, rs780094), glucose-6-phosphatase 2 (G6PC2) (rs1402837, rs560887), proprotein convertase subtilisin/kexin type 1 (PCSK1) (rs13179048, rs17085593, rs6235), protein phosphatase 1 regulatory subunit 3B (PPP1R3B) (rs4841132), and melatonin receptor 1B (MTNR1B) (rs1387153, rs7936247, rs10830963) were correlated with fasting plasma glucose levels in pregnant women [9]. Of these, PCSK1 is involved in the proteolytic activation of a variety of precursor proteins - including proinsulin, proglucagon-like peptide 1, and pro-opiomelanocortin [9]. Eleven SNPs related to GDM were identified in 8722 women from the United States and Denmark, among which eight were identified as rs7957197 (HNF1A), rs10814916 (GLIS3), rs3802177 (SLC30A8), rs9379084 (RREB1), rs34872471 (TCF7L2), rs7903146 (TCF7L2), rs11787792 (GPSM1), and rs7041847 (GLIS3). In addition, consistent with the results of previous studies, rs10830963 (MTNR1B), rs1387153 (MTNR1B), and rs4506565 (TCF7L2) were also associated with increased risk for GDM [10]. Most of the 11 identified SNPs were associated with pancreatic function, such as rs7957197, located in the intron region of HNF1A (which encodes a transcription factor needed to express GLUT1 and GLUT2 in pancreatic cells), and rs3802177, located in the intron region of SLC30A8 (which encodes a zinc transporter that is expressed only in secretory vesicles of $\beta$-cells and participates in the final stage of insulin biosynthesis and secretion) [10]. However, SLC30A8 overexpression in pancreatic $\beta$-cells was shown to increase the insulin secretion stimulated by glucose. GLIS3, a member of the GLI-similar zinc-finger protein family (with $5 \mathrm{C} 2 \mathrm{H} 2$-type zinc-finger domains) is highly expressed in pancreatic $\beta$-cells, and its variations relate to neonatal diabetes [10].

Kwak et al. [11] conducted two stages of GWAS in Korean women. In the first stage, 2.19 million genotyping or estimation markers were used to compare 468 women with GDM and 1242 women without GDM as controls [11]. According to the preset threshold of $p<2.0 \times 10^{-5}$, a total of nine SNP cues were associated with GDM, among which CDKAL1 (rs7754840) and MTNR1B (rs10830962) exhibited the strongest correlation with risk for GDM. In the second stage with 931 cases of GDM, the investigators chose 11 loci in 783 cases of the control group for further genotyping [11]. In the combined study of the two phases, they found, by using the gene loci from the second stage, that the first stage cues still showed a significant correlation between samples - with novel significantly related genetic loci, that is, IGF2BP2 (rs1470579) and insulin-degrading enzyme (IDE, rs10882066) [11]. A study of 218 Han Chinese women also identified 23 SNPs associated with GDM [12]. The SNPs manifested a dispersed distribution on 14 chromosomes, with only a few SNPs located on a specific chromosome. Most of these SNPs were not localized to specific genes, with only six SNPs located in the SYNPR, PTGIS, CDH18, and CTIF genes [12], these genes are involved in sugar metabolism-related pathways as revealed by enrichment of GO and KEGG pathway analyses. As an inherent component of synaptic vesicles, SYNPR plays a potential role in the synthesis and transport of neurotransmitters, and thus, may influence the signal-transduction process of glucose metabolism [12]. PTGIS, a member of the cytochrome P450 superfamily, is involved in prostaglandin 12 synthase (or prostacyclin synthase) and is closely tied to glucose and insulin levels in human endothelial cells [12]. CDH18 is one of the important cadherin genes located in the region of chromosome 5 , encodes type II classical cadherin, mediates calcium-dependent cell adhesion process, and may be involved in insulin-signal transduction through the wingless-type MMTV integration side family (Wnt)-signaling pathway [12].

Researchers using GWAS have identified several genes and loci associated with the occurrence and development of GDM in differ- 
ent geographic regions and ethnic groups and demonstrated that these genes were different for various ethnic groups. They also found, in their studies of genes and the underlying pathways involved, that the functions of these candidate genes primarily focused on glucose metabolism, insulin synthesis and secretion, and insulin-signal transduction.

\section{Genes Associated with Risk for GDM}

\section{Genes involved in $\beta$-cell function and insulin secretion}

The gene encoding cyclin-dependent kinase 5 regulatory subunit associated protein 1-like 1 (CDKAL1), is located in the 6p22.3 chromosomal region in humans [13]. CDKAL1 negatively regulates cyclin-dependent kinase 5 (CDK5) to affect insulin expression in pancreatic $\beta$-cells, inhibit insulin secretion, and transmit glucose-toxicity signals [14]. Additionally, CDKAL1 has been shown to be a methylthiotransferase that modifies the tRNA coding for lysine to enhance translation fidelity of transcripts, including those encoding proinsulin [15]. Researchers using a meta-analysis of five studies from Denmark, Korean, China, Malaysia and India - and involving a population of more than 7000 individuals - found that polymorphisms in CDKAL1 rs7754840 and rs7756992 were associated with an increased risk for GDM in Asian and Caucasian populations [14]. A nested case-control study of 321 participants with GDM and 316 controls in a Han Chinese population showed that the $G$ alleles of the CDKAL1 gene (i. e., rs4712527, rs7748720, rs9350276, and rs6938256) were all associated with a reduced risk of GDM; however, the risk alleles A, G, and G of rs9295478, rs6935599, and rs7747752 were all associated with an increased risk for GDM [16].

The cyclin-dependent kinase inhibitor 2A/2B (CDKN2A/2B) gene encodes 2 kinase inhibitors, $p 16^{\text {INK4a }}$ and $p 15^{\text {INK4b }}$, that inhibit cyclin-dependent kinases 4 (CDK4) and 6 (CDK6), which, in turn, regulate pancreatic $\beta$-cell function [17]. Overexpression of these two genes may reduce the proliferation of pancreatic $\beta$-islet cells, and CDKN2A/2B have been identified as T2DM candidate genes by GWAS [18]. Guo and co-works conducted a meta-analysis of 5996 women from Denmark, Korea, Spain, the U.S., and Poland, and found that individuals with the CDKN2A/2B rs10811661 T allele had a moderate tendency to develop GDM in general and showed a particular association in Asian and Caucasian populations [14]. Other contemporaneous workers uncovered an association between the rs7020996 risk allele T and reduced insulin sensitivity and increased insulin disposition in Han Chinese women [15].

Glucokinase (GCK), encoded by the GCK gene on chromosome $7 p 13$, belongs to the hexokinase family of proteins, and is a key regulatory enzyme in $\beta$-cells of the pancreas that catalyze glucose phosphorylation and regulate the release of insulin $[19,20]$. Case-control studies have shown that GCK rs4607517 and rs1799884 polymorphisms played a role in the risk for GDM in the Chinese population, and meta-analyses have also shown that rs 1799884 polymorphisms were associated with GDM in Caucasian and Asian populations [21]. In addition, the activity of GCK is regulated by the glucokinase regulatory protein (GCKRP), which is encoded by the glucokinase regulatory (GCKR) gene located on chromosome 2p23.3 and is involved in the synthesis of glycogen and triglycerides [14,22, 23]. A meta-analysis found that the GCKR rs780094 polymorphism was a risk factor for GDM in Caucasian populations [14], and the rs780094 C allele was shown to significantly increase the risk of developing GDM in the Caucasian population of Brazil and Asian population of Malaysia [19,24].

The potassium voltage-gated channel, KQT-like subfamily member1 (KCNQ1) gene located on chromosome 11p15 encodes the pore-forming alpha-subunit of the voltage-gated $\mathrm{K}^{+}$channel (KvLQT1) [20]. Insulin release from $\beta$-cells of the pancreas is controlled by an intricate interaction between ATP-sensitive potassium (KATP) channels, voltage-dependent potassium (Kv) channels, and voltage-dependent calcium channels. KCNQ1 expressed in the $\beta$-cells may therefore control insulin secretion by altering membrane potential and ion-channel function of pancreatic cells, thus affecting insulin production [25-27]. Two studies were conducted in Korea, involving a total of 1799 participants with GDM, which showed that the C alleles of KCNQ1 rs2237892 and rs2237895 or rs2074196 were associated with an increased risk of GDM [26, 28]. A meta-analysis involving Korean and Chinese populations also showed that rs2237892 with the $C$ allele increased the risk of GDM [29], while another study showed that the polymorphism of KCNQ1 rs2237895 was associated with increased GDM risk in Pakistan [27].

Melatonin receptor 1B (MTNR1B) is located on human chromosome $11 q 14.3$ and encodes MT2, one of the 2 high-affinity forms of the melatonin receptor [ 30,31$]$. MTNR1B is principally expressed in the retina, brain, and pancreatic cells; and is involved in the regulation of insulin release, circadian rhythms, and blood glucose homeostasis by modifying the melatonin-signaling pathway [32]. A recent meta-analysis that included the MTNR1B rs1387153, rs4753426, and rs10830963 loci in a case-control study with 23 participants of Caucasians origin and individuals in Asia and Mexico, findings indicated that the rs1387153, rs4753426, and rs 10830963 polymorphisms were associated with a widely regional susceptibility to GDM [33]. Furthermore, it was also shown that rs10830963 G allele polymorphism was significantly associated with GDM in Saudi, Hungarian, and Austrian women [31, 34].

The transcription factor 7-like 2 (TCF7L2) gene is located on chromosome 10q25, and the encoded TCF7L2 belongs to the high mobility group-box family as a transcription factor. This factor participates in the Wnt-signaling pathway, which can stimulate the proliferation of the $\beta$-cells of the pancreas and participate in the secretion of insulin by stimulating the production of glucagon-like peptide 1 (GLP-1) [35-38]. A meta-analysis by Chang et al. included 22 studies involving 8 SNPs of the TCF7L2 gene, of which rs12255372 and rs7901695 were significantly correlated with GDM; rs290487, rs11196205, and rs11196218 were only studied in a Han Chinese population, and rs290487 was significantly correlated with the risk of GDM, but not rs11196205. Subgroup analysis showed that rs 12255372 was not correlated with GDM in the Asian population and rs 7901695 was significantly correlated in all populations [35]. Moreover, rs7901695 polymorphism was independent of the risk for GDM in Poles and Brazilians [38, 39]. Another meta-analysis showed that rs7903146 was strongly associated with the risk for GDM in Asian and Caucasian populations [40], but that rs7903146 polymorphism was not associated with GDM in either Germany or Poland [36, 39]. Studies have also shown that the rs4506565 allele increased the risk of GDM in women in the United States and Denmark [10]. Rs290487, rs6585194, and rs7094463 
were associated with insulin resistance and insulin secretion in Chinese participants with GDM [41].

The vitamin D receptor (VDR) gene is localized on chromosome 12q13.11, and the encoded VDR, a member of the steroid/thyroid hormone receptor family that functions as a transcriptional activator of numerous genes, is essential for vitamin $D$ activity in target tissues $[42,43]$. Vitamin D exerts its effect via regulation of extracellular calcium levels in the $\beta$-cells, thereby promoting insulin secretion [44]. Zhu et al. investigated the relationships among vitamin D concentration, VDR gene polymorphism, and GDM risk in Chinese women; they found that vitamin $D$ concentrations in women were not significantly associated with the risk of GDM. However, women with the rs 1544410 CT or rs 731236 GA genotype exhibited a higher risk of developing GDM [45]. Contemporaneously, several studies showed that the genotypic frequencies for VDR rs 19735810 TT and CT were significantly elevated in Turkish women with GDM [42]; that the rs7975232 CC and rs731236 TT genotypes increased the risk of GDM in Iranian women [44]; and that the rs 739837 polymorphism was associated with fasting glucose concentration in Chinese women [43]. In contrast, other studies have shown that rs 10735810 was not associated with GDM in Brazilian women [46].

Insulin-like growth factor 2 mRNA-binding protein 2 (IGF2BP2) belongs to the insulin-like growth factor 2 (IGF2) mRNA-binding protein family and is encoded by the IGF2BP2 gene located on chromosome 3q27.2 [47]. IGF2BP2 is highly expressed in pancreatic islets and plays an important role in embryonic growth and development, as well as in insulin secretion and in the regulation of pancreatic $\beta$-cell function [48]. Previous studies have shown that the IGF1BP2 rs4402960 polymorphism is significantly associated with GDM in Korean and Chinese women $[49,50]$. However, in contradistinction, recent case-control studies and meta-analyses shown that rs4402960 polymorphism was not associated with GDM risk in either Asian or Caucasian women [51]. Due to these author' small sample size and limited study populations, their conclusion needs to be supported by additional research results.

The primary roles of the gene-encoding proteins reviewed above are in regulating islet cell proliferation and insulin secretion. Studies have shown that polymorphisms of these genes were significantly correlated with the occurrence of GDM, and that this correlation has been verified in several geographic regions. However, the studies have only focused on the polymorphisms of a few genetic loci, and whether GDM is related to the polymorphisms of additional gene loci requires further examination.

\section{Genes involved in glucose metabolism and insulin resistance}

Adiponectin (ADIPOQ) genes located on chromosome 3q27.3 are mainly expressed in adipose tissue and encode proteins that are major components of adiponectin [52], which is considered an insulin-sensitizing adipokine with anti-inflammatory effects. Adiponectin is involved in reducing insulin resistance by promoting the receptor/post-receptor insulin signaling and reducing gluconeogenesis [53]. In the meta-analyses of ADIPOQ (rs2241766, rs 1501299, and rs266729) polymorphisms, the researchers found that rs2241766 was significantly associated with GDM in Asian women, but that rs 1501299 and rs266729 polymorphisms were not associated with GDM in the enrolled population [54]. Another meta-analysis showed that rs266729 increased the risk of GDM in Asian and European populations but reduced the risk of GDM in the U.S. population [55].

The calpain-10 (CAPN10) gene, which is located within the non-insulin dependent diabetes mellitus 1 (NIDDM1) region on chromosome $2 \mathrm{q} 37.3$, is one of the earliest genes discovered to be associated with T2DM [56]. The CAPN10 gene encodes for a $\mathrm{Ca}^{2+}$-requiring non-lysosomal cysteine protease, calpain-10, that is expressed in the pancreas, skeletal muscle, and adipocytes. Studies have suggested that calpain-10 plays a role in glucose transporter 4 translocation to the cell membrane, regulation of pancreatic glucose-induced insulin secretion, and pancreatic $\beta$-cell apoptosis [57]. Expression of CAPN10 has been shown to be associated with T2DM, and it is closely correlated with blood glucose and insulin secretion [58]. Researchers in Mexico found that the genotypic frequencies of rs3792267, rs2975760, rs5030952, and indel19 showed no significant differences between women with GDM and normal controls, although the glucose levels in women with an indel-19 polymorphism in the $3 R / 3 R$ genotype were significantly elevated relative to women with the $3 R / 2 R$ genotype [57]. Two studies entailing meta-analyses on the relationship between CAPN10 rs3842570, rs3792267, rs2975760, and rs5030952 polymorphisms and GDM in Asian and Caucasian populations showed that only the rs 3842570 and rs3792267 polymorphisms were associated with GDM in Asian populations [40,56]. However, in the study of Han Chinese women by Zhang et al., the polymorphisms of rs 2975760 and rs 3792267 were not significantly correlated with the occurrence of GDM, and only slightly correlated with condition severity [58].

The hexokinase domain containing 1 (HKDC1) gene is located near the hexokinase 1 (HK1) gene on chromosome 10q22.1 and is widely expressed in liver and $\beta$-islet cells [59]. The encoded HKDC1 protein possesses both a glucose-binding domain and an ATP-binding site in its $\mathrm{COOH}$-terminal region, indicating its hexokinase activity and its principal role in mediating glucose metabolism $[9,60]$. Kanthimathi et al. analyzed the HKDC1 polymorphisms rs 10762264, rs4746822, rs2394529, and rs9645501 in 500 women with GDM in India, and found that the AA genotype of rs 10762264 , TT genotype of rs 4746822, and CC genotype of rs2394529 all increased the risk for GDM by over 1.6-fold [59]. Another research group also found that the rs 4746822 polymorphism was significantly correlated with 2-hour plasma glucose in Han Chinese women [61].

The macrophage migration inhibitory factor (MIF) gene is positioned at 22q11.23 [62]. Although MIF molecules are not considered to belong to the cytokine family [63], most participate in the critical regulatory functions of macrophage and lymphocyte activation in innate and adaptive immune responses, and the MIF gene has been identified to be a novel candidate gene for T2DM [64]. When MIF levels are attenuated, glucose pathways are improved due to reduced peripheral insulin resistance and the regulatory effects of glucocorticoids $[62,65]$. Further, Yilmaz et al. found that serum insulin levels and MIF concentrations in Caucasian women with GDM in Turkey were significantly higher than in healthy control women, suggesting that MIF may play a role in the development of GDM [65]. Studies have shown that fasting blood glucose 
and insulin levels and insulin resistance in pregnant women with the GG genotype MIF rs 1007888 were significantly higher than in pregnant women with other genotypes [63]. The number of rs755622 C allele carriers in GDM women was also significantly higher than in the normal control group, suggesting that this allele may be a risk factor for GDM [64] Aslani et al. also found a high frequency of the rs 1007888 GG genotype and a significant association with preconception obesity in Indian women with GDM [62].

The insulin receptor substrate-1 (IRS-1) gene, located on chromosome $2 q 36.3$, is a member of the IRS protein substrate family [66], is a substrate of the insulin receptor tyrosine kinase and a participant in insulin signaling and is involved in insulin resistance [67]. The G972R (rs1801278) polymorphism of IRS- 1 that is located between the two possible tyrosine-phosphorylation sites for $\mathrm{Pl}-3 \mathrm{ki}$ nase $\mathrm{p} 85$ subunit binding has also previously been shown to be associated with T2DM [66]. The aforementioned meta-analyses included 5 studies in Asian and Caucasian populations that showed a significantly increased risk for GDM due to the presence of the IRS1 rs1801278 T allele $[67,68]$.

The beta-adrenergic receptors (ADRBs) consist of the $\beta 1$ adrenergic receptor (ADRB1), $\beta 2$ adrenergic receptor (ADRB2), and $\beta 3$ adrenergic receptor ( $\beta 3-A R, A D R B 3)$ [69]. The ADRB3 gene is located on human chromosome $8 p 11.23$, encoding ADRB3, which is primarily expressed in the adipose tissue. It is a member of the $G$ protein-coupled receptor superfamily that mediates heat generation and fat decomposition stimulated by catecholamines and occupies a significant position in the activation of energy storage and utilization of lipid decomposition and internal environmental stability of glucose [69-72]. Therefore, the gene expression defect caused by an ADRB3 mutation is the initial factor in insulin resistance [70]. Guan et al., in a meta-analysis of five studies that included 1239 participants with GDM from four Caucasian and one Asian region, demonstrated that the Trp64Arg (rs4994) polymorphism in ADRB3 was associated with GDM susceptibility in Caucasian populations in Europe [73]. Another study found that the rs201607471 CT genotype was significantly correlated with an increased risk for GDM in a Han Chinese population [70].

The alfa2-Heremans-Schmid glycoprotein (AHSG) gene is located on chromosome 3q27.3, and encodes fetuin-A, a serum glycoprotein expressed in the liver $[74,75]$. Studies have shown that fetuin-A induces insulin resistance by inhibiting insulin receptor tyrosine kinase activity and signal transduction in muscle and liver tissue and that it is also associated with the accumulation of fat in liver cells, which is a risk factor for diabetes [75, 76]. A study of an American population showed that serum fetuin-A concentrations were significantly higher in participants with gestational diabetes relative to normal controls, and that the allelic frequency of AHSG rs4918 G was also associated with GDM risk [76]. Another group explored the correlation between rs 2248690 and rs 4918 polymorphisms and GDM in 83 women with GDM in Turkey, and their results showed that the genotypic distribution of rs 2248690 was not different between GDM peoples and the control group; but that the GG genotype of rs4918 might exert protective effect against the occurrence of GDM [75].

Interleukin-10 (IL-10) is an important anti-inflammatory cytokine produced by activated $\mathrm{T}$ cells, monocytes, and B cells, and its coding gene is located on chromosome 1q32.1 [77]. The gene composition of the IL10 promoter appears to then determine the secretion levels of IL-10 [78]. Several studies have suggested that an imbalance in the production of anti-inflammatory and pro-inflammatory cytokines during pregnancy may lead to reduced levels of inflammation and insulin resistance [79]. In a study from Taiwan, that included 72 women with GDM and 100 healthy controls, investigators analyzed the relationships between the IL-10 gene polymorphism rs1800896, rs 1800871, rs1800872, and rs3021094 and GDM susceptibility [77]. Their results showed increased insulin resistance in individuals with allele $A$ of rs3021094, and that IL-10 levels in the AA genotype carriers were significantly higher than in the CC genotype carriers [77]. Another study, in a Polish population, showed higher weights and augmented BMI during pregnancy in women with the rs1800872AA genotype [79].

These results suggest that glucose metabolism- and insulin resistance-related gene polymorphisms are associated with the incidence of GDM. The correlations, however, varied with respect to different geographic regions and populations, and the susceptibility-gene loci were not exactly identical. Therefore, it is necessary to expand the study populations to confirm the role(s) of these genes in the development of GDM.

\section{Genes involved in oxidative stress and other pathways}

The angiotensin I-converting enzyme (ACE) gene is located on 17q23.3. ACE converts angiotensin I to angiotensin II and is an important component in the renin-angiotensin system (RAS), which may be associated with insulin resistance and the occurrence of T2DM [80]. The ACE gene has an insertion/deletion (I/D) polymorphism that is due to the presence or deletion of a 287-bp fragment within intron 16 , and the $\mathrm{D}$ allele was found to be associated with an increased risk for microvascular and macrovascular complications in T2DM [81]. Aggarwal et al. found that ACE A240T, C1237T, G2350A, and I/D were significantly correlated with GDM participants in India and that individuals with the haplotype TTAI with these polymorphisms had a 185 -fold increased risk of developing GDM compared with normal controls. Khan et al. also found that the I/D polymorphism of the ACE gene's Alu sequence was associated with the risk of GDM in Indian women [82, 83]. However, in the latest published study, the researchers did not find statistically significant differences in the gene frequency of G298A and I/D polymorphisms between Indian women with GDM and women without GDM [80]. Intriguingly, this correlation between ACE polymorphisms and GDM has not been reported in any population outside of India.

The human paraoxonase 1 (PON1) protein is coded by the PON1 gene located on the long arm of chromosome 7 (7q21.3), and most common polymorphisms are localized to the coding region: a leucine (L) to methionine (M) substitution at position 55 (L55M, rs854560), and a glutamine (Q) to arginine (R) substitution at position 192 (Q192R, rs662) [84]. PON1 is a serum/plasma high-density lipoprotein-binding enzyme primarily expressed in the liver that protects HDL, low-density lipoprotein (LDL), endothelial cells, and intimal macrophages from oxidative damage by catalyzing the hydrolysis of organic phosphates and lipid peroxides [85-87]. Lipoprotein oxidation is the key to the development of diabetic macrovascular (atherosclerosis) and microvascular disease, and the tox- 


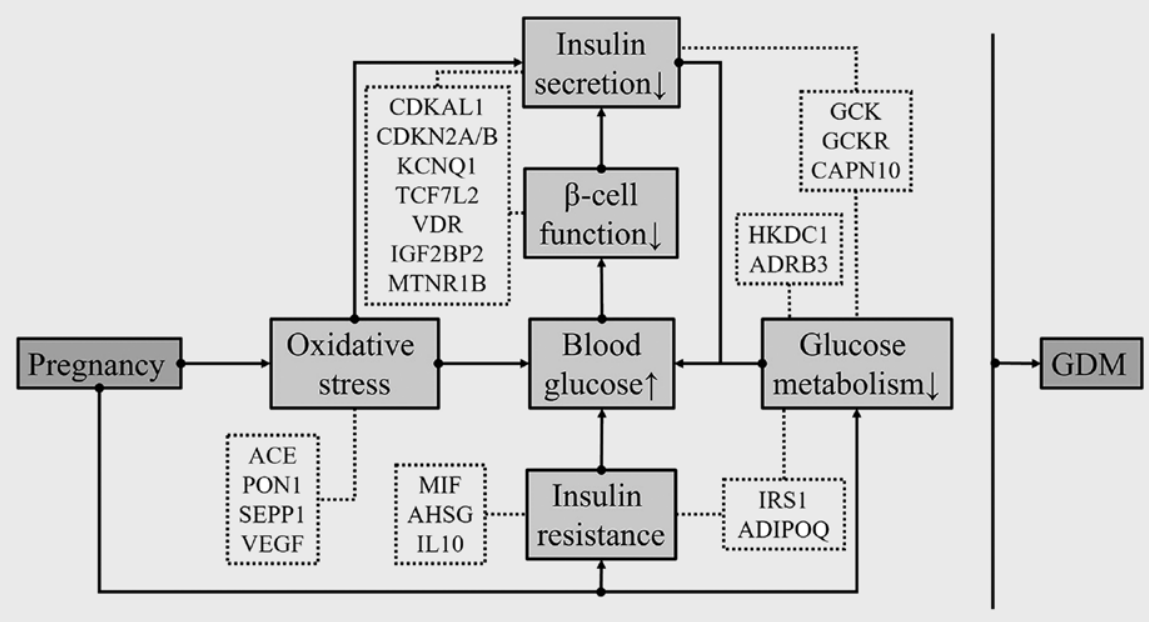

Fig. 1 Mechanisms and genes related to the development of GDM.

- Table 1 Genetic polymorphisms associated with the risk of gestational diabetes mellitus in different regions.

\begin{tabular}{|c|c|c|c|c|}
\hline No. & Gene & SNP & Populations & Author [Ref] \\
\hline \multirow[t]{2}{*}{1} & \multirow[t]{2}{*}{ GCKR } & \multirow[t]{2}{*}{ rs780094 } & Brazilian & Anghebem-Oliveira [19] \\
\hline & & & Malaysian & Jamalpour [24] \\
\hline \multirow[t]{4}{*}{2} & \multirow[t]{4}{*}{ KCNQ1 } & \multirow[t]{2}{*}{ rs2237892 } & Chinese & Ao [29] \\
\hline & & & Korean & Kwak [11] \\
\hline & & \multirow[t]{2}{*}{ rs2237895 } & Pakistani & Fatima [27] \\
\hline & & & Korean & Kwak [26] \\
\hline \multirow[t]{2}{*}{3} & \multirow[t]{2}{*}{ MTNR1B } & \multirow[t]{2}{*}{ rs10830963 } & Saudi & Alharbi [34] \\
\hline & & & Polish & Tarnowski [48] \\
\hline \multirow[t]{2}{*}{4} & \multirow[t]{2}{*}{ TCF7L2 } & \multirow[t]{2}{*}{ rs7903146 } & Mexican & Reyes-López [97] \\
\hline & & & Greek & Pappa [89] \\
\hline \multirow[t]{2}{*}{5} & \multirow[t]{2}{*}{ VDR } & \multirow[t]{2}{*}{ rs731236 } & Iranian & Rahmannezhad [44] \\
\hline & & & Chinese & Zhu [45] \\
\hline \multirow[t]{2}{*}{6} & \multirow[t]{2}{*}{ IGF2BP2 } & \multirow[t]{2}{*}{ rs4402960 } & Korean & Chon [49] \\
\hline & & & Chinese & Wang [50] \\
\hline \multirow[t]{2}{*}{7} & \multirow[t]{2}{*}{ ADIPOQ } & \multirow[t]{2}{*}{ rs2241766 } & Chinese & Feng [52] \\
\hline & & & Iranian & Takhshid [98] \\
\hline \multirow[t]{2}{*}{8} & \multirow[t]{2}{*}{ MIF } & \multirow[t]{2}{*}{ rs1007888 } & Iranian & Aslani [62] \\
\hline & & & Chinese & Zhan [63] \\
\hline \multirow[t]{2}{*}{9} & \multirow[t]{2}{*}{ IRS1 } & \multirow[t]{2}{*}{ rs1801278 } & Italian & Fallucca [71] \\
\hline & & & Saudi & Alharbi [66] \\
\hline \multirow[t]{2}{*}{10} & \multirow[t]{2}{*}{ AHSG } & \multirow[t]{2}{*}{ rs4918 } & Turkish & Akbaba [91] \\
\hline & & & Americans & Tariq [76] \\
\hline \multirow[t]{2}{*}{11} & \multirow[t]{2}{*}{ PON1 } & \multirow[t]{2}{*}{ rs662 } & Saudi & Al-Hakeem [85] \\
\hline & & & Greek & Рappa [89] \\
\hline
\end{tabular}

SNP: Single-nucleotide polymorphism. 
icity of oxidized lipoprotein to retinal capillary endothelial cells and perivascular cells is significant [87]. Zhou et al. found that lactonase activity and PON1 levels were both increased in female Chinese participants with GDM, and that women with the PON1 Q192R RR genotype exhibited lower PON1 lactonase activities and normalized lactonase activity relative to other genotypes [88]. The PON1 Q192R gene polymorphism has been shown to be associated with GDM in Turkish, Greek, and Saudi women; the RR genotypes and R alleles were significantly associated with increased GDM risk in Greek women, while the L55M MM polymorphism was only associated with GDM risk in Turkish women [85, 86, 89].

The selenoprotein P (SEPP1) gene is located on chromosome $5 \mathrm{p} 12$, and the protein contains a selenocysteine residue [90]. It is primarily synthesized by the liver, and acts as an antioxidant to attenuate oxidative stress, transport aelenium, and downregulate the insulin-signaling pathway via AMP-activated protein kinase (AMPK) inactivation in vivo $[90,91]$. In a case-control study, the Turkish women with the SEPP1 rs13154178 AA genotype manifested significantly higher fasting blood glucose levels than women with other genotypes [91].

In addition, studies have shown that the leucine-rich repeat and Ig domain containing 2 (LINGO2) rs824248 AT and TT genotypes located on chromosome 9p21 can significantly increase the risk for GDM [92]. The protein encoded by the SOS Ras/Rac guanine nucleotide exchange factor 1 (SOS1) gene localized to $2 \mathrm{p} 22$ is a guanine nucleotide-exchange factor of RAS proteins [93]. Data from Taiyuan, China showed that women with the rs7598922 GA/AA genotype and rs3099968 AG and rs1010859 GG genotypes exhibited a lower risk of developing GDM compared to women with other genotypes [94]. The vascular endothelial growth factor (VEGF) gene located on chromosome 6p21 encodes VEGF (an endothelial cell-specific mitogen), which is the most critical angiogenic growth factor involved in increasing vascular permeability [92, 95]. Dong's study confirmed that the VEGF genotypes rs2146323 CC and rs3025039 CC significantly reduced the risk of GDM in Chinese women [96].

This section summarized the relationship between GDM and gene polymorphisms with respect to oxidative stress-related endothelial injury and other related functions. However, the studies only entailed a limited number of geographic areas and population groups, and therefore the specific underlying mechanism(s) and correlations between genetic polymorphisms and GDM need to be confirmed by additional in-depth analyses.

\section{Conclusion}

GDM is one of the most common complications in pregnancy and can exert short- and long-term impacts on pregnant women and fetuses. There are many risk factors associated with the occurrence of GDM, of which genetic susceptibility is critically important. There are several GDM risk genes (GCKR, KCNQ1, MTNR1B, etc.) with the functions of glucose metabolism, insulin synthesis and secretion, as well as insulin-signal transduction, that have been identified by the GWAS. Besides, investigators have used case-control studies to explore the susceptibility genes involved in GDM, and found that several genes (TCF7L2, VDR, IGF2BP2, etc.) subserving multiple functions such as $\beta$-cell function, insulin secretion, peripheral in- sulin resistance, glucose metabolism, and oxidative stress, confer genetic susceptibility to GDM (see > Fig. 1, > Table 1, and Tables S1, S2). However, due to the genetic complexity of GDM, it is difficult to deduce its underlying molecular mechanism(s) from a relatively limited sample size. Therefore, further studies of additional samples, different genetic structures, and environmental conditions found in different regions of the world are required to elucidate the pathogenesis of GDM in greater detail.

\section{Funding Information}

This work was supported by National Key R \& D Program of China (grant number: 2018YFC1004300), National Natural Science Foundation Project of China (grant number: 81670844), the Key Project of Guizhou Provincial Science and Technology Department (grant number: QKH-JC-2019-1464), the Excellent Talent Support Program of Guizhou Provincial Education Department (grant number: QJH-KY-2017-077), the Science and Technology Foundation of Guizhou Province (grant number: QKH-PTRC-2017-5733-003, QKH-PTRC-2018-5772-042), the Program for Excellent Young Talents of Zunyi Medical University (18-ZY-001), the science and technology program project of Zunyi (ZSKH-HZ-2020-35), and the project of Scientific Research Foundation for Postgraduates of Guizhou Province (grant number: QJH-YJSCXJH-2019-094).

\section{Conflict of Interest}

The authors declare that they have no conflict of interest.

\section{References}

[1] Aydin H, Celik O, Yazici D et al. Prevalence and predictors of gestational diabetes mellitus: a nationwide multicentre prospective study. Diabetic Med 2019; 36: 221-227

[2] Xie K, Zhang Y, Wen J et al. Genetic predisposition to gestational glucose metabolism and gestational diabetes mellitus risk in a Chinese population. J Diabetes 2019; 11: 869-877

[3] Popova PV, Klyushina AA, Vasilyeva LB et al. Effect of gene-lifestyle interaction on gestational diabetes risk. Oncotarget 2017; 8: 112024-112035

[4] Mclntyre HD, Catalano P, Zhang C et al. Gestational diabetes mellitus. Nat Rev Dis Primers 2019; 5: 47

[5] Moen GH, Sommer C, Prasad RB et al. Mechanisms in endocrinology. Epigenetic modifications and gestational diabetes: a systematic review of published literature. Eur J Endocrinol 2017; 176: R247-R267

[6] Plows JF, Stanley JL, Baker PN et al. The pathophysiology of gestational diabetes mellitus. Int J Mol Sci 2018; 19

[7] Zhang H, Wang Q, He S et al. Ambient air pollution and gestational diabetes mellitus: A review of evidence from biological mechanisms to population epidemiology. Sci Total Environ 2020; 719: 137349

[8] Hirschhorn JN, Daly MJ. Genome-wide association studies for common diseases and complex traits. Nat Rev Genet 2005; 6: 95-108

[9] Hayes MG, Urbanek M, Hivert MF et al. Identification of HKDC1 and BACE2 as genes influencing glycemic traits during pregnancy through genome-wide association studies. Diabetes 2013; 62: 3282-3291 
[10] Ding M, Chavarro J, Olsen S et al. Genetic variants of gestational diabetes mellitus: a study of 112 SNPs among 8722 women in two independent populations. Diabetologia 2018; 61: 1758-1768

[11] Kwak SH, Kim SH, Cho YM et al. A genome-wide association study of gestational diabetes mellitus in Korean women. Diabetes 2012; 61: 531-541

[12] Wu NN, Zhao D, Ma W et al. A genome-wide association study of gestational diabetes mellitus in Chinese women. J Matern Fetal Neonatal Med 2019; 1-8

[13] Steinthorsdottir V, Thorleifsson G, Reynisdottir I et al. A variant in CDKAL1 influences insulin response and risk of type 2 diabetes. Nat Genet 2007; 39: 770-775

[14] Guo F, Long W, Zhou W et al. FTO, GCKR, CDKAL1 and CDKN2A/B gene polymorphisms and the risk of gestational diabetes mellitus: a meta-analysis. Arch Gynecol Obstet 2018; 298: 705-715

[15] Mei ], Liao S, Liu Y et al. Association of variants in CDKN2A/2B and CDKAL1 genes with gestational insulin sensitivity and disposition in pregnant Han Chinese women. J Diabetes Investig 2015; 6: 295-301

[16] Wang K, Chen Q, Feng Y et al. Single nucleotide polymorphisms in CDKAL1 gene are associated with risk of gestational diabetes mellitus in Chinese population. J Diabetes Res 2019; 3618103

[17] Tarnowski M, Malinowski D, Safranow K et al. CDC123/CAMK1D gene rs12779790 polymorphism and rs10811661 polymorphism upstream of the CDKN2A/2B gene in women with gestational diabetes. J Perinatol 2017; 37: 345-348

[18] Wang X, Li W, Ma L et al. Association study of the miRNA-binding site polymorphisms of CDKN2A/B genes with gestational diabetes mellitus susceptibility. Acta Diabetol 2015; 52: 951-958

[19] Anghebem-Oliveira MI, Webber S, Alberton D et al. The GCKR gene polymorphism rs 780094 is a risk factor for gestational diabetes in a Brazilian population. J Clin Lab Anal 2017; 31

[20] Ao D, Zhao Q, Song JY et al. The association of the glucokinase rs4607517 polymorphism with gestational diabetes mellitus and its interaction with sweets consumption in Chinese women. Public Health Nutr 2020; 1-7

[21] Han X, Cui H, Chen X et al. Association of the glucokinase gene promoter polymorphism - 30G >A (rs1799884) with gestational diabetes mellitus susceptibility: A case-control study and meta-analysis. Arch Gynecol Obstet 2015; 292: 291-298

[22] Lin Z, Wang Y, Zhang B et al. Association of type 2 diabetes susceptible genes GCKR, SLC30A8, and FTO polymorphisms with gestational diabetes mellitus risk: a meta-analysis. Endocrine 2018; 62: 34-45

[23] Tarnowski M, Malinowski D, Pawlak K et al. GCK, GCKR, FADS1, DGKB/ TMEM195 and CDKAL1 gene polymorphisms in women with gestational diabetes. Can J Diabetes 2017; 41: 372-379

[24] Jamalpour S, Zain SM, Mosavat M et al. A case-control study and meta-analysis confirm glucokinase regulatory gene rs 780094 is a risk factor for gestational diabetes mellitus. Gene 2018; 650: 34-40

[25] Dixit G, Dabney-Smith C, Lorigan GA. The membrane protein KCNQ1 potassium ion channel: Functional diversity and current structural insights. Biochim Biophys Acta Biomembr 2020; 1862: 183148

[26] Kwak SH, Kim TH, Cho YM et al. Polymorphisms in KCNQ1 are associated with gestational diabetes in a Korean population. Horm Res Paediatr 2010; 74: 333-338

[27] Fatima SS, Chaudhry B, Khan TA et al. KCNQ1 rs2237895 polymorphism is associated with gestational diabetes in Pakistani women. Pak J Med Sci 2016; 32: 1380-1385

[28] Shin HD, Park BL, Shin H] et al. Association of KCNQ1 polymorphisms with the gestational diabetes mellitus in Korean women. J Clin Endocrinol Metab 2010; 95: 445-449

[29] Ao D, Wang H], Wang LF et al. The rs2237892 polymorphism in KCNQ1 influences gestational diabetes mellitus and glucose levels: a case-control study and meta-analysis. PLoS One 2015; 10: e0128901
[30] Tarnowski M, Malinowski D, Safranow K et al. MTNR1A and MTNR1B gene polymorphisms in women with gestational diabetes. Gynecol Endocrinol 2017; 33: 395-398

[31] Rosta K, Al-Aissa Z, Hadarits O et al. Association study with 77 SNPs confirms the robust role for the rs $10830963 / G$ of MTNR1B variant and identifies two novel associations in gestational diabetes mellitus development. PLoS One 2017; 12: e0169781

[32] Zhan Y, Li C, Gao Q et al. Association between the rs 4753426 polymorphism in MTNR1B with fasting plasma glucose level and pancreatic beta-cell function in gestational diabetes mellitus. Genet Mol Res 2015; 14: 8778-8785

[33] Jia G, Gao Y, Li C et al. Effects of MTNR1B genetic variants on individual susceptibility to gestational diabetes mellitus: a meta-analysis. Am J Perinatol 2020; 37: 607-612

[34] Alharbi KK, Al-Sulaiman AM, Shedaid KMB et al. MTNR1B genetic polymorphisms as risk factors for gestational diabetes mellitus: a case-control study in a single tertiary care center. Ann Saudi Med 2019; 39: 309-318

[35] Chang S, Wang Z, Wu L et al. Association between TCF7L2 polymorphisms and gestational diabetes mellitus: A meta-analysis. J Diabetes Investig 2017; 8: 560-570

[36] Fritsche L, Sarief M, Wagner R et al. Genetic variation in TCF7L2 rs7903146 and history of GDM negatively and independently impact on diabetes-associated metabolic traits. Diabetes Res Clin Pract 2018; 146: $251-257$

[37] Potasso L, Perakakis N, Lamprinou A et al. Clinical impact of the TCF7L2 gene rs 7903146 type 2 diabetes mellitus risk polymorphism in women with gestational diabetes mellitus: impaired glycemic control and increased need of insulin therapy. Exp Clin Endocrinol Diabetes 2020; 128: 663-666

[38] Anghebem-Oliveira MI, Martins BR, Alberton D et al. Type 2 diabetes-associated genetic variants of FTO, LEPR, PPARg, and TCF7L2 in gestational diabetes in a Brazilian population. Arch Endocrinol Metab 2017; 61: 238-248

[39] Michalak-Wojnowska M, Gorczyca-Siudak D, Gorczyca T et al. Association between rs 7901695 and rs 7903146 polymorphisms of the TCF7L2 gene and gestational diabetes in the population of Southern Poland. Ginekol Pol 2016; 87: 745-750

[40] Hou Z, Li M, Cao Y. TCF7L2, CAPN10 polymorphisms are associated with gestational diabetes mellitus (GDM) risks: A meta-analysis. Gynecol Endocrinol 2017; 33: 399-404

[41] Ye D, Fei Y, Ling Q et al. Polymorphisms in TCF7L2 gene are associated with gestational diabetes mellitus in Chinese Han population. Sci Rep 2016; 6: 30686

[42] Apaydin M, Beysel S, Eyerci N et al. The VDR gene Fokl polymorphism is associated with gestational diabetes mellitus in Turkish women. BMC Med Genet 2019; 20: 82

[43] Wang Y, Wang O, Li W et al. Variants in vitamin D binding protein gene are associated with gestational diabetes mellitus. Medicine (Baltimore) 2015; 94: e1693

[44] Rahmannezhad G, Mashayekhi F], Goodarzi MT et al. Association between vitamin $D$ receptor Apal and Taql gene polymorphisms and gestational diabetes mellitus in an Iranian pregnant women population. Gene 2016; 581: 43-47

[45] Zhu B, Huang K, Yan S et al. VDR Variants rather than early pregnancy vitamin $D$ concentrations are associated with the risk of gestational diabetes: the Ma'anshan Birth Cohort (MABC) Study. J Diabetes Res 2019; 8313901

[46] Siqueira TW, Araujo Junior E, Mattar R et al. Assessment of polymorphism of the VDR gene and serum vitamin $D$ values in gestational diabetes mellitus. Rev Bras Ginecol Obstet 2019; 41: 425-431 
[47] Rao P, Wang H, Fang $\mathrm{H}$ et al. Association between IGF2BP2 Polymorphisms and Type 2 diabetes mellitus: a case-control study and meta-analysis. Int J Environ Res Public Health 2016; 13

[48] Tarnowski M, Bujak J, Kopytko P et al. Effect of FTO and IGF2BP2 gene polymorphisms on duration of pregnancy and Apgar scores in women with gestational diabetes. J Obstet Gynaecol 2019; 39: 151-156

[49] Chon S], Kim SY, Cho NR et al. Association of variants in PPARgam$\mathrm{ma}(2)$, IGF2BP2, and KCNQ1 with a susceptibility to gestational diabetes mellitus in a Korean population. Yonsei Med J 2013; 54: 352-357

[50] Wang Y, Nie M, Li W et al. Association of six single nucleotide polymorphisms with gestational diabetes mellitus in a Chinese population. PLoS One 2011; 6: e26953

[51] Liu ], Song G, Zhao G et al. Lack of association between IGF2BP2 rs4402960 polymorphism and gestational diabetes mellitus: A case-control study, meta-analysis and trial sequential analysis. Biosci Rep 2020; 40

[52] Feng $\mathrm{Y}$, Jiang $C D$, Chang $A M$ et al. Interactions among insulin resistance, inflammation factors, obesity-related gene polymorphisms, environmental risk factors, and diet in the development of gestational diabetes mellitus. J Matern Fetal Neonatal Med 2019; 32: 339-347

[53] de Gennaro G, Palla G, Battini L, Simoncini T et al. The role of adipokines in the pathogenesis of gestational diabetes mellitus. Gynecol Endocrinol 2019; 35: 737-751

[54] Huang LT, Wu SL, Liao X et al. Adiponectin gene polymorphisms and risk of gestational diabetes mellitus: a meta-analysis. World J Clin Cases 2019; 7: 572-584

[55] Bai Y, Tang L, Li L et al. The roles of ADIPOQ rs266729 and MTNR1B rs10830963 polymorphisms in patients with gestational diabetes mellitus: a meta-analysis. Gene 2020; 730: 144302

[56] Cui ], Xu X, Yin S et al. Meta-analysis of the association between four CAPN10 gene variants and gestational diabetes mellitus. Arch Gynecol Obstet 2016; 294: 447-453

[57] Castro-Martinez AG, Sanchez-Corona J, Vazquez-Vargas AP et al. Association analysis of calpain 10 gene variants/haplotypes with gestational diabetes mellitus among Mexican women. Cell Mol Biol (Noisy-le-grand) 2018; 64: 81-86

[58] Zhang X, Shi C, Wei L et al. The association between the rs2975760 and rs3792267 single nucleotide polymorphisms of calpain 10 (CAPN10) and gestational diabetes mellitus. Med Sci Monit 2019; 25: 5137-5142

[59] Kanthimathi S, Liju S, Laasya D et al. Hexokinase domain containing 1 (HKDC1) gene variants and their association with gestational diabetes mellitus in a South Indian population. Ann Hum Genet 2016; 80: 241-245

[60] Khan MW, Priyadarshini M, Cordoba-Chacon J et al. Hepatic hexokinase domain containing 1 (HKDC1) improves whole body glucose tolerance and insulin sensitivity in pregnant mice. Biochim Biophys Acta Mol Basis Dis 2019; 1865: 678-687

[61] Tan YX, Hu SM, You YP et al. Replication of previous genome-wide association studies of HKDC1, BACE2, SLC16A11 and TMEM163 SNPs in a gestational diabetes mellitus case-control sample from Han Chinese population. Diabetes Metab Syndr Obes 2019; 12: 983-989

[62] Aslani S, Hossein-nezhad A, Maghbooli Z et al. Genetic variation in macrophage migration inhibitory factor associated with gestational diabetes mellitus and metabolic syndrome. Horm Metab Res 2011; 43: 557-561

[63] Zhan Y, Li C, Chen J et al. Association between macrophage migration inhibitory factor rs1007888 and GDM. Genet Mol Res 2015; 14: 797-804

[64] Li C, Qiao B, Qi W et al. Association of macrophage migration inhibitory factor polymorphisms with gestational diabetes mellitus in Han Chinese women. Gynecol Obstet Invest 2016; 81: 84-89
[65] Yilmaz O, Kucuk M, Kebapcilar L et al. Macrophage migration-inhibitory factor is elevated in pregnant women with gestational diabetes mellitus. Gynecol Endocrinol 2012; 28: 76-79

[66] Alharbi KK, Khan IA, Abotalib Z et al. Insulin receptor substrate-1 (IRS-1) Gly927Arg: correlation with gestational diabetes mellitus in Saudi women. Biomed Res Int 2014; 146495

[67] Zhang Y, Sun CM, Hu XQ et al. Relationship between melatonin receptor $1 \mathrm{~B}$ and insulin receptor substrate 1 polymorphisms with gestational diabetes mellitus: a systematic review and meta-analysis. Sci Rep 2014; 4: 6113

[68] Wu L, Cui L, Tam WH et al. Genetic variants associated with gestational diabetes mellitus: a meta-analysis and subgroup analysis. Sci Rep 2016; 6: 30539

[69] Luo Z, Zhang T, Wang S et al. The Trp64Arg polymorphism in beta3 adrenergic receptor (ADRB3) gene is associated with adipokines and plasma lipids: a systematic review, meta-analysis, and meta-regression. Lipids Health Dis 2020; 19: 99

[70] Jia H, Pan Y, Wang Y et al. Beta-3 adrenergic receptor gene polymorphisms are associated with gestational diabetes mellitus in a Chinese population. Medicine (Baltimore) 2019; 98: e17258

[71] Fallucca F, Dalfra MG, Sciullo E et al. Polymorphisms of insulin receptor substrate 1 and beta3-adrenergic receptor genes in gestational diabetes and normal pregnancy. Metabolism 2006; 55: 1451-1456

[72] Tsai PJ, Ho SC, Tsai LP et al. Lack of relationship between beta3-adrenergic receptor gene polymorphism and gestational diabetes mellitus in a Taiwanese population. Metabolism 2004; 53: 1136-1139

[73] Guan L, Cui X, Zhou H. Meta-analysis of the association between the Trp64Arg polymorphism of the beta-3 adrenergic receptor and susceptibility to gestational diabetes mellitus. J Obstet Gynaecol 2018; 38: 172-176

[74] Kalabay L, Cseh K, Pajor A et al. Correlation of maternal serum fetuin/ alpha2-HS-glycoprotein concentration with maternal insulin resistance and anthropometric parameters of neonates in normal pregnancy and gestational diabetes. Eur J Endocrinol 2002; 147: 243-248

[75] Akbas H, Kahraman S, Sak S et al. Minor variant of AHSG gene 767C $>C$ polymorphism may decrease the risk of gestational diabetes mellitus. J Obstet Gynaecol 2020; 40: 303-307

[76] Tariq A, Asghar A, Alam F et al. AHSG rs4918 polymorphism poses a weak predisposition to insulin resistance during pregnancy. J Pak Med Assoc 2018; 68: 698-701

[77] Kang J, Liu CH, Lee CN et al. Novel interleukin-10 gene polymorphism is linked to gestational diabetes in Taiwanese population. Front Genet 2019; 10: 89

[78] Montazeri S, Nalliah S, Radhakrishnan AK. Is there a genetic variation association in the IL-10 and TNF alpha promoter gene with gestational diabetes mellitus? Hereditas 2010; 147: 94-102

[79] Majcher S, Ustianowski P, Tarnowski M et al. IL-1beta and IL-10 gene polymorphisms in women with gestational diabetes. J Matern Fetal Neonatal Med 2019; 1-6

[80] Mirfeizi M, Hasanzad M, Sattari M et al. Association of eNOS and ACE gene polymorphisms as a genetic risk factor in gestational diabetes in Iranian women. J Diabetes Metab Disord 2018; 17: 123-127

[81] Kajantie E, Rautanen A, Kere J et al. The effects of the ACE gene insertion/deletion polymorphism on glucose tolerance and insulin secretion in elderly people are modified by birth weight. J Clin Endocrinol Metab 2004; 89: 5738-5741

[82] Khan IA, Jahan P, Hasan Q et al. Angiotensin-converting enzyme gene insertion/deletion polymorphism studies in Asian Indian pregnant women biochemically identifies gestational diabetes mellitus. J Renin Angiotensin Aldosterone Syst 2014; 15: 566-571

[83] Aggarwal P, Agarwal N, Das N et al. Association of polymorphisms in angiotensin-converting enzyme gene with gestational diabetes mellitus in Indian women. Int ] Appl Basic Med Res 2016; 6: 31-37 
[84] Aydemir B, Behice Serinkan Cinemre F, Cinemre $H$ et al. Clinical significance of PON1 L55M, Q192R and I102V polymorphisms and their association with prostate cancer risk in Polish men. Pol J Pathol 2020; 71: 55-61

[85] Al-Hakeem MM, Abotalib Z, Alharbi KK et al. Relationship between the paraoxonase 1 gene glutamine 192 to arginine polymorphism and gestational diabetes mellitus in Saudi women. Clin Biochem 2014; 47 : 122-125

[86] Aydemir B, Behice Serinkan Cinemre F, Cinemre $\mathrm{H}$ et al. Paraoxonase 1 (PON1) Q192R and L55M polymorphisms, lipid profile, lipid peroxidation and lipoprotein-a levels in Turkish patients with pregnancy-related disorders. Gynecol Endocrinol 2019; 35: 417-421

[87] Rosta V, Trentini A, Passaro A et al. Sex difference impacts on the relationship between paraoxonase-1 (PON1) and type 2 diabetes. Antioxidants (Basel) 2020; 9: 683

[88] Zhou M, Liu XH, Liu QQ et al. Lactonase activity, status, and genetic variations of paraoxonase 1 in women with gestational diabetes mellitus. J Diabetes Res 2020; 3483427

[89] Pappa KI, Gazouli M, Anastasiou E et al. The Q192R polymorphism of the paraoxonase-1 (PON1) gene is associated with susceptibility to gestational diabetes mellitus in the Greek population. Gynecol Endocrinol 2017; 33: 617-620

[90] Gharipour M, Ouguerram K, Nazih EH et al. Effect of single nucleotide polymorphisms in SEPS1 and SEPP1 on expression in the protein level in metabolic syndrome in subjects with cardiovascular disease. Mol Biol Rep 2019; 46: 5685-5693
[91] Akbaba G, Akbaba E, Sahin C et al. The relationship between gestational diabetes mellitus and selenoprotein-P plasma 1 (SEPP1) gene polymorphisms. Gynecol Endocrinol 2018; 34: 849-852

[92] Su T, Ren Q, Lu Y et al. A genetic variant in LINGO2 contributes to the risk of gestational diabetes mellitus in a Chinese population. J Cell Physiol 2019; 234: 7012-7018

[93] Baltanas FC, Zarich N, Rojas-Cabaneros JM et al. SOS GEFs in health and disease. Biochim Biophys Acta Rev Cancer 2020; 1874: 188445

[94] Chen Q, Yang H, Feng $Y$ et al. SOS1 gene polymorphisms are associated with gestational diabetes mellitus in a Chinese population: Results from a nested case-control study in Taiyuan, China. Diab Vasc Dis Res 2018; 15: 158-161

[95] Krishnasamy S, Ravi V, Rajaraman B et al. Role of VEGF165b/ VEGFTOTAL ratio in gestational diabetes mellitus. Gynecol Endocrinol 2019; 35: 811-814

[96] Dong PP. Association of vascular endothelial growth factor expression and polymorphisms with the risk of gestational diabetes mellitus. J Clin Lab Anal 2019; 33: e22686

[97] Reyes-López R, Pérez-Luque E, Malacara J. Metabolic, hormonal characteristics and genetic variants of TCF7L2 associated with development of gestational diabetes mellitus in Mexican women. Diabetes Metab Res Rev 2014; 30: 701-706

[98] Takhshid M, Haem Z, Aboualizadeh F. The association of circulating adiponectin and $+45 \mathrm{~T} / \mathrm{G}$ polymorphism of adiponectin gene with gestational diabetes mellitus in Iranian population. J Diabetes Metab Disord 2015; 14: 30 